\title{
Survey on Car Detection Techniques Using Aerial Images
}

\author{
Azharoddin Inamdar ${ }^{1}$, Zameer Farooqui ${ }^{2}$ \\ ${ }^{1}$ Department of E\&TC, Aditya Engineering College, Beed, India, \\ ${ }^{2}$ Professor, Department of E\&TC, Aditya Engineering College, Beed, India,
}

\begin{abstract}
Now a days Car detection techniques are developing and existing techniques are improving to get better accuracy results. This methods mostly used in traffic monitoring, speed management and also in military applications. Aerial view has the advantage of providing a better perspective of the area being covered. So in this area experts make use of the aerial images or videos taken from aerial vehicles. Detection of cars can be either from the dynamic aerial imagery, wide area motion imagery or the images can be of low resolution and static in nature. The purpose of this paper is to provide a survey of research related to car detection techniques with better accuracy level and the application of car detection techniques for traffic management and other applications.
\end{abstract}

Keywords: Car detection, feature extraction, scale invarient feature transform (SIFT). Histogram of oriented gradient (HOG), Support Vector machine (SVM).

\section{Introduction}

In the last few years, there are number of vehicles are increases on the roads and also in parking lots. So that some problems are generated, such as traffic jams and parking problems which are mainly found in big city areas. So optimization of the urban traffic planning and management is necessary. Now a day in the classes of objects, a research community gives particular attention to the cars. Also in the field of object detection, the most discussed issue is determination of the number of cars on the roads or in parking lots. Aerial surveillance provides increased monitoring results in case of fast-moving targets because spatial area coverage is greater. Thus aerial surveillance is supplement for ground plane surveillance systems. One of the main topics in intelligent aerial surveillance is vehicle detection and tracking. The difficulties involved in the aerial surveillance include the camera motions such as panning, tilting and rotation. Also the different camera heights largely affect the detection results. Vision based techniques is one of the most common approach to analyze vehicles from images or videos. The view of cars will vary according to the camera positions, lighting conditions and background situations. The existing car detection techniques are based on a large variety of techniques. This paper provides a survey on the existing methods which to an extent overcomes the disadvantages. One method utilizes color transformation in case of still images and an approach tends to utilize wide area motion imagery. Another technique seems to provide detection in low resolution strategy and another based on Histogram of Oriented Gradient (HOG) features.

\section{Literature Review}

Hierarchical model proposed by Hinz and Baumgartner [1] which describes different levels of details of vehicle features and detection method based on cascade classifiers has the disadvantage of lots of miss detections. Luo-wei tsai, Jun-wei hsieh, Kuo-chin fan et al. [2] proposed a novel detection method using color transform model. Hansen et al. [3] defined an affine model for the operation of motion detection. Daniel A. lavigne et al. [4] makes use of aerial images which has up to an average $11.2 \mathrm{~cm} /$ pixel resolution. Xuelong Li et al. [5] introduce an extension of the HOG features overcoming the disadvantages. The newly develops technique is known as boosting HOG feature.

\section{Detection Techniques}

\subsection{Detection using normalized color and edge map}

The detection procedure is done in different stages. In the first stage a color transformation model is used to easily identify the vehicle (car) pixels from backgrounds .The technique which is adopted for this is dimensionality reduction. Beginning of the technique follows the collection of several thousands of training images. For this all colors of input pixels are projected on the color space. The classification is carried out by means of a Bayesian classifier. The detected pixel corresponds to a car. Different car hypothesis are generated from each detected pixels. The verification of hypothesis is done by edges, coefficients of wavelet transform and corners. An optimal vehicle classifier can be formed by using proper weights from a set of training samples. By using this method cars can be very robustly and accurately verified and detected from static images.

\subsection{Robust vehicle detection in low resolution aerial imagery}

In this methodology a Scale Invariant Feature Transform (SIFT) is used. This technology effectively generates all the keypoints.SVM is also utilized to classify these key points to an extent. Scale Invariant Feature Transform (SIFT) is a technique which is invariant to rotation, scaling, illumination changes etc. The task is effective for the natural outdoor images. The advantage which attracts the authors is that the output is affected by noise and distortion to a very low extend. SVM or Support Vector Machine is a supervised learning algorithm. This algorithm greatly aids in classifying

\section{Volume 5 Issue 2, February 2016}




\section{International Journal of Science and Research (IJSR) \\ ISSN (Online): 2319-7064}

Index Copernicus Value (2013): 6.14 | Impact Factor (2014): 5.611

the generated key points in the learned way. Hyper plane concept is used to separate the key points to separate classes. For this purpose the features has to map to a new space and on this space only the hyper plane separation is done. The next and final stage of the technique is the usage of Affinity Propagation algorithm (AP) on the separated key points. The main task in the method of clustering is the finding out of a center for the clusters. Cluster centers have to be developed in such a way that the instances or data should attain stability. Stability in the sense it should not transfer from one cluster to another. This reduces the error rate

\subsection{Context-driven moving car detection in wide area motion imagery}

Detection of moving cars in wide area motion imagery has many promising applications nowadays. As the need increases the challenges in this area also keeps on increasing. A step wise framework is insisted which include the motion detection firstly. After the task the detection of cars is done by the trained classifier. The second step does the most prominent feature in the work that is the usage of the scene context information. The next step deals with the usage of the shape and gradient distribution [6]. In the motion detection phase stabilization operation is seemed to be carried out which considerably eliminates the unwanted motions from a dynamic video sequence. Background subtraction operation follows the operation. The next phase which comes in detail is binary classification. The actual task performed in the phase is the car detection. Classification is carried out by support vector machine along with considering the features such as HOG and size. HOG features means histogram of oriented gradient which represents the object contours to an extent. Size features are considered for distinguishing the cars from the false alarms as cars have consistent size and shape representations. 4. Linear SVM classification using boosting HOG features for car detection in low altitude airborne videos: In this method an extension of the HOG features overcoming the disadvantages. The newly develops technique is known as boosting $\mathrm{HOG}$ feature. Adboost classifier is used for this boosting HOG features. These features are utilized in training a linear SVM classifier. This framework utilizes low-altitude airborne platform. The linear
SVM classification is used for the final car classification. The method is divided into two parts which includes feature description and classifier training. The HOG features have the disadvantage of having high dimensionality. Boosting HOG features overcome the disadvantage by reducing the dimensionality. Hog features are actually considered as a histogram. The bins of the histogram are representatives of the weak classifiers. Ad boost training is required to translate the weak to strong classifiers. These strong classifiers are on the way combined to construct a feature vector. The final stage contributes by training the SVM classifier for the final output of car detection.

\section{Comparison}

The four different techniques in car detection are summarized to table 1 which helps to distinguish between the advantages and disadvantages of the techniques which utilizes different input images and videos taken at different scenarios. To clearly illustrate the differences between the different techniques

available the different parameters such as technique, false alarm rate, training samples. Technique clearly depicts the method used for detection. False alarm rate is the important parameter which indicates the performance of the method. Inspite of the SVM technique the area deals with another technique such as SIFT which considerably reduces the false alarm rate. The actual accuracy rates of all the different techniques are illustrated in table 2 . The performance measures such as accuracy and false alarm rate are compared for the research purpose. Analysis of the techniques shows that context information contributes most for getting greatest detection results. False rate also seems to reduce to a considerable amount. We insist on increasing the accuracy of the car detection.

\section{Figures and Tables}

The fig. 1 shows aerial car image captured by aerial vehicle. These types of aerial car images are used for car detection techniques.

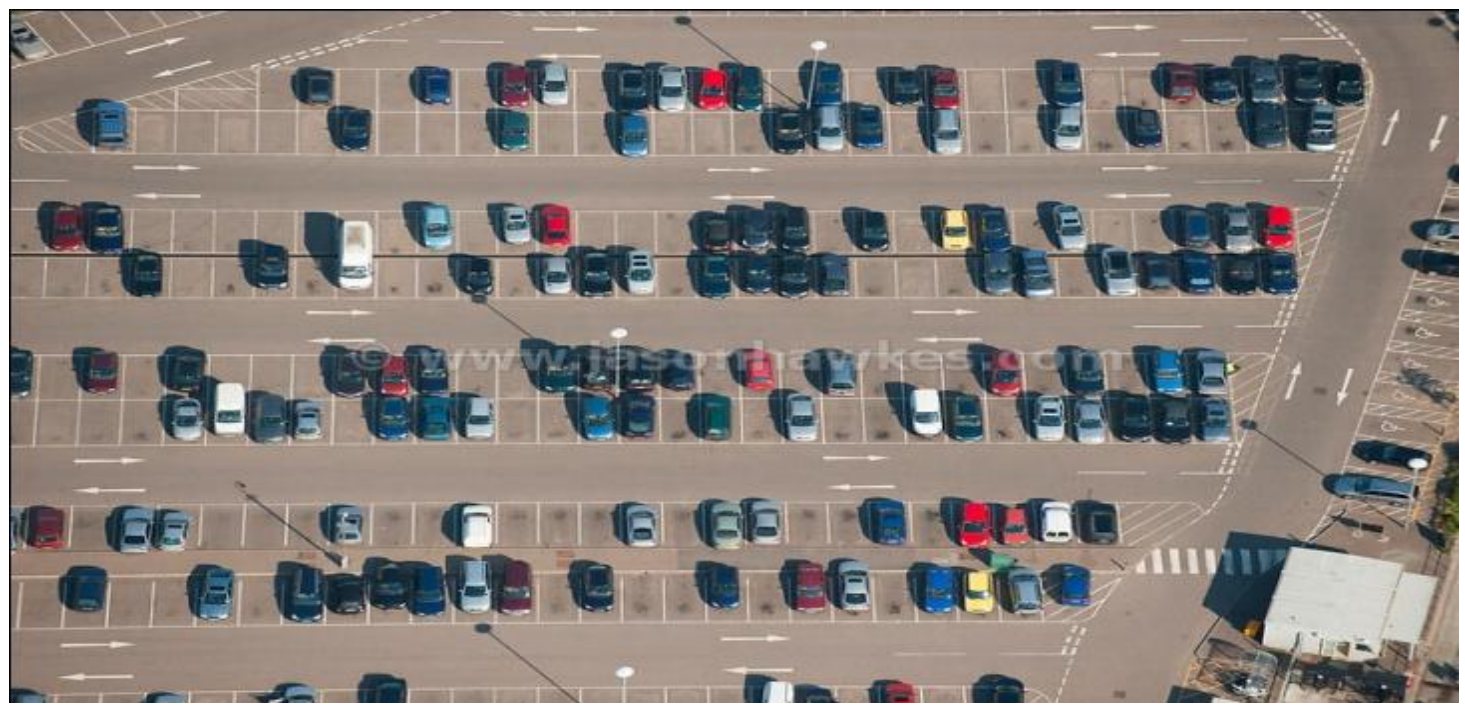

Figure 1: Sample aerial car image

Volume 5 Issue 2, February 2016 


\section{International Journal of Science and Research (IJSR)}

ISSN (Online): 2319-7064

Index Copernicus Value (2013): 6.14 $\mid$ Impact Factor (2014): 5.611

Table: 1 Comparison of four different car detection methods

\begin{tabular}{|l|l|l|l|}
\hline Topic & Technique & False alarm rate (FAR) & Training Samples \\
\hline $\begin{array}{l}\text { Normalized Color } \\
\text { and edge map }\end{array}$ & $\begin{array}{l}\text { Color transform } \\
\text { model }\end{array}$ & $\begin{array}{l}\text { Due to RBF (Radial Basis } \\
\text { Function) network }\end{array}$ & $\begin{array}{l}\text { Large number } \\
\text { required }\end{array}$ \\
\hline $\begin{array}{l}\text { Low resolution } \\
\text { aerial imagery }\end{array}$ & SIFT with SVM & SIFT reduces FAR & $\begin{array}{l}\text { Very less } \\
\text { number }\end{array}$ \\
\hline $\begin{array}{l}\text { Context driven } \\
\text { moving car detection }\end{array}$ & $\begin{array}{l}\text { SVM and HOG and } \\
\text { context extraction }\end{array}$ & $\begin{array}{l}\text { Reduces Context } \\
\text { extraction. }\end{array}$ & $\begin{array}{l}\text { Uses the CLIF } \\
\text { dataset }\end{array}$ \\
\hline $\begin{array}{l}\text { Boosting HOG } \\
\text { features }\end{array}$ & $\begin{array}{l}\text { Boosting HOG with } \\
\text { linear SVM }\end{array}$ & $\begin{array}{c}\text { High false alarm } \\
\text { rate }\end{array}$ & $\begin{array}{l}\text { More than 3 hours } \\
\text { of video are taken }\end{array}$ \\
\hline
\end{tabular}

Table: 2 Detection accuracy and false alarm rate using four different detection methods.

\begin{tabular}{|l|c|c|}
\hline \multicolumn{1}{|c|}{ Detection Method } & Accuracy & False alarm rate \\
\hline Normalized Color and edge map & $94 \%$ & $8.50 \%$ \\
\hline Low resolution aerial imagery & $92 \%$ & $7.26 \%$ \\
\hline Context driven moving car detection & $95 \%$ & $7.17 \%$ \\
\hline Boosting HOG features & $90 \%$ & $10 \%$ \\
\hline
\end{tabular}

\section{Conclusion}

The paper presents the four car detection techniques. The main aim of this survey is to explore an ideal approach of nearly no false alarms and high detection accuracy. For an ideal method number of training samples should also be considerably low. The method using normalized color and edge map requires a large number of training samples and it is observed that the method using the SIFT considerably reduces it. The other two methods mainly make use of HOG features for the detection and SVM for classification but the simple HOG has the disadvantage of high dimensionality which reduces the detection speed and accuracy. These methods specifically rely on the CLIF (Columbus Large Image Format) dataset and also on the urban traffic videos. The survey points to the fact that car detection using dynamic Bayesian networks can be applied on a broader extent and the false detections is considerably reduced and it works well by taking only a small training dataset which is a great advantage. It is observed that using background removal and enhanced edge and corner detection has increased the detection accuracy which is appreciable.

\section{Acknowledgements}

The author would like to thank Mr. Zameer Farooqui for valuable support and guidance for completing this survey paper work.

\section{References}

[1] S. Hinz and A. Baumgartner, "Vehicle detection in aerial images uses generic features, grouping, and context," in Proc. DAGM-Symp.,Sep.2001,vol.2191, Lecture Notes in Computer Science, pp. 45-52.

[2] Luo-wei tsai, Jun-wei hsieh, member, IEEE, and Kuochin fan, member, IEEE, "Vehicle detection using normalized color and edge map", IEEE Transactions On Image Processing, vol. 16, no. 3, March 2007.
[3] M. Hansen, P. Anadan, K. Dana, G. van de Wal, P. Burt, "Real-timescene stabilization and Mosaic Construction", Proc of IEEE CVPR, 1994, 54-62)

[4] Samir Sahli, Yueh Ouyang, Yunlong Sheng, Daniel A. lavigne "Robust vehicle detection in low-resolution aerial imagery" a Image Science group.

[5] Xianbin Cao, Changxia Wu, Pingkun Yan, Xuelong Li3 "Linear SVM classification using boosting hog features for vehicle detection in low-altitude airborne videos" University of Science and Technology of China.

[6] Xinchu Shi1, Haibin Ling, Erik Blasch, Weiming Hul "Context-Driven Moving Vehicle Detection in Wide Area Motion Imagery" National Laboratory of Pattern Recognition, Institute of Automation, Beijing, China Department of Computer and Information Science, Temple University, Philadelphia, USA Air Force Research Lab, USA

[7] S.Tuermer,j.Leitloff ,P.Reinartz ,U.Stilla “Automatic vehicle detection in aerial image sequences of urban areas using 3d hog features" paparoditis n., pierrotdeseillignym., mallet c., tournaire o. (eds), iaprs, vol. xxxviii, part $3 b$ - saint-mandé, France, September 13, 2010.

[8] J. Y. Choi and Y. K. Yang, "Vehicle detection from aerial images using local shape information," Adv. Image Video Technol., vol. 5414, Lectures Notes in Computer Science, pp. 227-236, Jan. 2009

[9] Jie Zhou, senior member, IEEE, Dashan Gao, and David Zhang, senior member, IEEE "Moving vehicle detection for automatic traffic monitoring" IEEE Transactions On Vehicular Technology, vol. 56, no. 1, January 2007.

[10]Line Eikvil, Lars Aurdal and Hans Koren "Classification-based vehicle detection in high resolution satellite images" Norwegian Computing Center.

[11]Lowe, D., "Distinctive Image features from Scale Invariant Key points," International Journal of Computer Vision. Papers 60 (2), 91-110 (2004). 